\title{
Maintaining Functional Module Integrity in Sensor Networks
}

\author{
Kanthakumar Pongaliur, Chen Wang, Li Xiao \\ Department of Computer Science and Engineering \\ Michigan State University \\ East Lansing, MI 48823 \\ Contact: \{pongaliu,wangchen, lxiao\} @ cse.msu.edu
}

\begin{abstract}
Security is one of the top concerns when sensors are deployed in hostile environments for military applications. Attacks on sensor nodes can be categorized into two types: attacks through radio signals to alter the functionalities of sensors and physical attacks; which may completely damage sensors. We show that the former is easier to launch and more disguised, thus more hazardous. The layered framework proposed in this paper is specially designed to defend against the first type of attacks. Different from precious reputation-based approaches or data mining technologies which usually incur large communication and computation overhead, our solution only involves localized computation which is less intensive, thus can be implemented on-board with little burden on current MICA2 platforms. Besides identifying malicious nodes, our solution can also recover the normal functionalities of sensors, which have been altered by malicious radio signals.
\end{abstract}

Keywords- integrity; security; sensor networks;

\section{INTRODUCTION}

It is a crucial task to ensure sensors' security because sensors are intended to be deployed in hostile environments for some military applications, where sensors are directly exposed to attacks by adversary. On the other hand, it is necessary to reprogram sensors to satisfy the dynamically changing application requirements. This has to be automatically finished in wireless fashion because sensors are deployed to inaccessible areas in large volumes. The wireless reprogramming feature makes sensors more vulnerable because it can be easily taken advantage by attackers. The security requirements and the vulnerability make the security mechanism an essential part in sensor designs. However, the limited resources available in sensors make it a challenging task to implement on-board security mechanisms using encryption and authentication, which usually involve intensive computations in traditional approaches.

Realizing the urgency of securing sensors, a lot of research (TinySec, SPIN, TinyPK and SERP) has been devoted to this area. Most of the work is focused on providing security and integrity to transmission data, while less work is dedicated on protecting sensor nodes themselves; especially protect sensors from attacks initiated from wireless channels, which convert sensors to malicious nodes by reprogramming them through radio signals. Sensors reprogrammed with malicious intent can attack the entire network as follows: (1) take over a node and listen to the data being transmitted through the node; (2) introduce stale, corrupt data into the network; (3) destroy the node by depleting the node of its resources such as energy in a

This work was supported in part by the US National Science Foundation under grants CCF-0325760 and CCF-0514078. subversive manner; (4) or even consume the energy of the entire network through intentional broadcast.

Compared with the attacks that physically destroy [16] or alternate sensors, the attacks initiated from wireless channel may be more dangerous because of several reasons stated below:

- It is convenient to attack sensors through radio signals, because it is not required to physically access and occupy sensor nodes. It can be achieved even without the knowledge of sensors exact locations. Moreover, the attack can be initiated to large number of sensors in a cost effective fashion.

- It is harder to detect attacks initiated from wireless signals. Because it can be finished more quickly than physical damage, it is more difficult to catch the flying attacks. Unlike the physical attack, which may completely destroy sensors' functionalities, the attack initiated from wireless signals only alters the functionalities of sensors. The altered sensors with malicious intent will try to camouflage themselves by behaving normally at most times.

To identify malicious nodes, a reputation-based approach [7] has been proposed to assign reputation to the nodes based on the votes of neighbors. This achieves a high degree of success in filtering the bad data propagated by the malicious entity. Similar to the reputation-based approach, data mining technologies are suggested to statistically analyze sensors' activities and find out malicious nodes, which have abnormal behaviors. Such approaches usually involve heavy overhead including large communications to collect data and intensive computations to analyze data. Also it has a tradeoff between the high probability of identifying malicious nodes and the false-positive report. In this paper, we propose a layered framework to recognize malicious nodes, which have been reprogrammed using wireless radio signals. Unlike the mining approach, our solution can find out malicious nodes mainly depending on the localized computation on individual sensors and avoid broadcast messages. Also the computation overhead is small, thus it can be implemented in current MICA2 sensor platform.

The architecture of our framework is a layered structure with a middleware layer that is strongly bound to the operating system of TinyOS. In our proposed framework, a sensor node consists of four parts: the functional module, security middleware, the TinyOS and the hardware platform. Here, only functional module can be reprogrammed by radio signals to satisfy different applications requirements. Both the security middleware layer and TinyOS are hard-coded into the 
hardware and can only be changed by plugging wired cables. The security middleware layer is responsible for providing a secure environment for the execution of the various mobile agents. The middleware also acts as an interface between the application layer and the operating system.

We use mobile agents to query the status of the functional module and also recover the functional module in case of detection of its malfunction. Mobile agents have been defined as follows in [11]. "Mobile agents are programs that can migrate from host to host in a network, at times and to places of their own choosing. The state of the running program is saved, transported to the new host, and restored, allowing the program to continue where it left off." The advantage of having a mobile agent compared to a process migration system is the agents are independent entities and can make decisions by themselves, while process migration depends on the system to make decisions.

In our approach, the correctness and integrity of functional module is based on the underlying security middleware layer. Such a layered methodology provides a secure framework for sensors, since the security of more vulnerable functional module is built on the basis of middleware layer, which has to be attacked physically by restarting sensors or plugging in extra cables. Such a kind of attack is much easier to be detected.

The organization of the paper is as follows: In Section 2 we describe the attack model. Section 3 depicts the architectural framework and the working model. Then we discuss the detailed security and overhead analysis in Section 4. Related work follows as Section 5 and we have the conclusion and future work in Section 6.

\section{ATTACK MODEL}

We define the attack model in this section. We categorize attacks on sensor nodes into two types: (1) the attacks initiated from radio signals which try to reprogram sensor with malicious intent; (2) the physical attacks which completely take over sensors by plugging in wired cables. Accordingly, we divide sensor nodes into two parts: (1) the first part is the functional module which can be reprogrammed through radio signals; (2) the second part consists of security middleware and TinyOS, which are hard-coded in the hardware and can only be modified by plugging in cables.

By reprogramming sensor nodes through radio signals, an attacker can change the functional module and launch an attack with one or more of the following aims:

- Listen to the data being transmitted through the node

- Introduce stale/ corrupt data into the network

- Act as a sink and discard all the data passing through it

- Deplete the node of its resources.

- Keep transmitting garbage data; thereby depleting energy of entire network or occupying the communication channel.

Besides the attack activities above, a malicious node will try its best to disguise itself by periodically sending useful data, thus making the reputation-based approaches or data mining techniques harder to recognize its malicious intent.
In this paper, we only deal with the first type of attack, i.e. the attacks initiated through radio signals and can only change the functional module. Therefore, our work is based on the assumption that the lower layer of security middleware is secure and reliable. Such a layered concept makes the security of the entire sensor node easier to be approached, since modification of security middleware layer requires more attacking efforts and can be easier to detect. There has been some work to address such kinds of physical attacks. We are also working on solutions to further detect physical attacks, which requires either power cycle or plugging extra cables.

\section{ARCHITECTURE FRAMEWORK AND WORKING MODEL}

The architecture of the proposed framework is shown in Figure 1. We have the sensor node (MICA2 mote) running the TinyOS. We also have the TinySec module included as part of the operating system. On top of the operating system runs the middleware layer, providing the interface to the functional module.

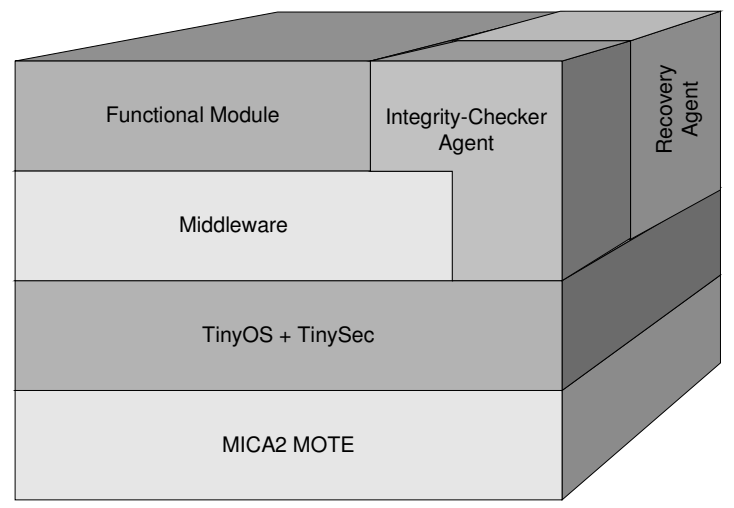

Figure 1: Architecture

As seen in Figure 1, the functional module rests on top of the middleware layer. This module is responsible for providing all the different application specific functions. The functional module should be designed such that we can do a reinstallation if it is corrupted. Hence it is recommended that its design needs to be highly modular for ease of transport using the recovery agents.

The middleware layer provides the suitable environment for the execution of the mobile agents. Also it may allow direct but restricted access to kernel for certain kinds of mobile agents. A resultant requirement is the security of the middleware, which gains paramount importance.

The base station, also called the sink is the central governing authority. It is responsible for checking the functional integrity of the node. We make two assumptions regarding the base station. The first assumption is that it has enormous resources including energy, memory, processing power and secondly it is secure against attacks. These assumptions are legit, since the number of base-stations will be few compared to the numerous sensor nodes. Hence the base stations can be considered to be 
powerful and secure. The base station has two modules, which are classified on the basis of their functionalities:

- Sensor Network Management Module (SNMM): The core functionality of this module is to manage the integrity checker agent (ICA). This requires it to generate an initialization vector, which is sent to the sensor node in the ICA. SNMM also keeps track of the expected message authentication code (called function authentication code in our system- FAC) from each node. It is also needed to know the counter value, which will be used to check if the node has undergone a restart since the previous check. In summary, the SNMM runs a simple database to keep track of the following information:

o Node-Id

o Counter value

o Type of counter algorithm

o Initialization Vector (2 bytes)

- Expected Function Authentication Code

- Recovery Module (RM): As the name suggests, this module is responsible for generating the recovery agent when the SNMM identifies a malfunctioning or corrupt functional module in a sensor node. It manages the recovery agents and is responsible for reinstallation of the functional module in the sensor node.

We discuss the security process in the following three subsections:

\section{A. Integrity Check Process}

The SNMM module in the base station creates the ICA at regular but random intervals of time. The ICA includes a unique 2 bytes initialization vector as discussed above. This ICA is sent to the corresponding sensor node, where the middleware provides the environment for the execution of the ICA. This process is shown in Figure 2.

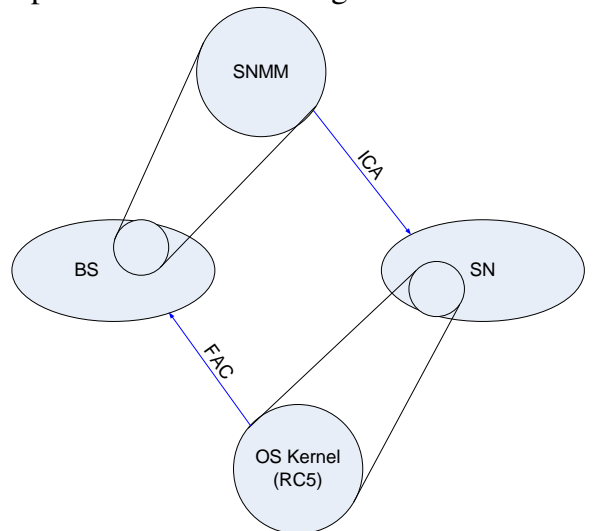

Figure 2: Integrity Check Process

After the checking process, the ICA returns to the base station with the FAC and the counter value. The sensor node then compares the FAC and the counter values with the expected FAC and expected counter values respectively. It gives rise to four scenarios, which is depicted in Figure 3.

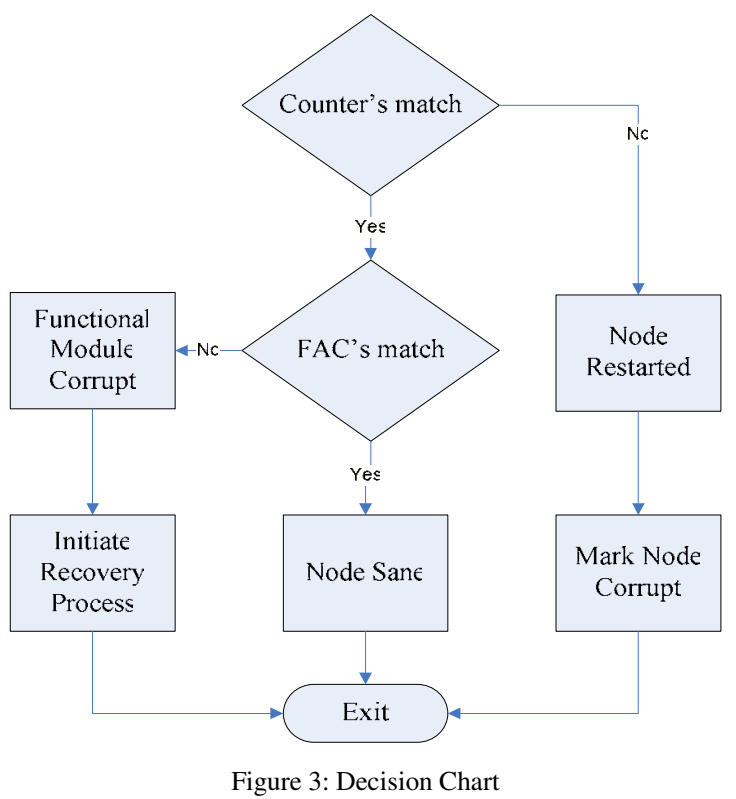

If both the function authentication codes match, then the functional module is marked as sane. If the two FAC's do not match, while the counter values match, the SNMM initiates the recovery process through the recovery module as shown in Figure 5.

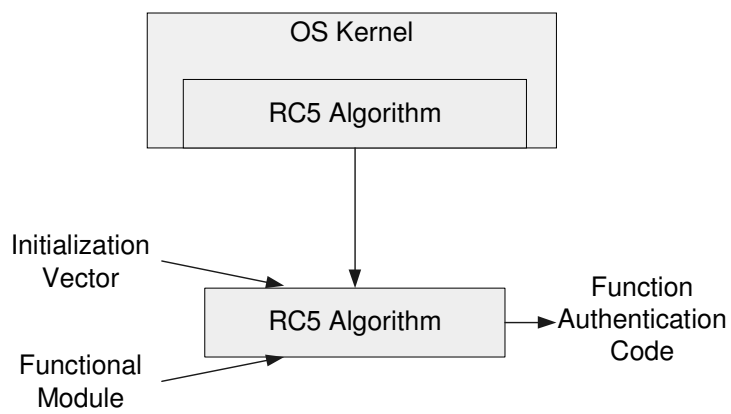

Figure 4: Function Authentication Code Generation

\section{B. FAC Generation Process}

The FAC generation process is shown in Figure 4. The initialization vector from the ICA and the entire functional module is given as input to the RC5 algorithm, which is already implemented as part of the TinySec module. The output of this process is an encrypted form of functional module and the function authentication code. We discard the encrypted form and care about only the FAC. This FAC is carried back to the base station by the ICA. This works on the principle that the message (the functional module in this case) and a unique IV will generate a unique FAC when encrypted using the RC5 algorithm [10].

As mentioned earlier, on receipt of a non-matching FAC, the recovery process is initiated as in Figure 5. 


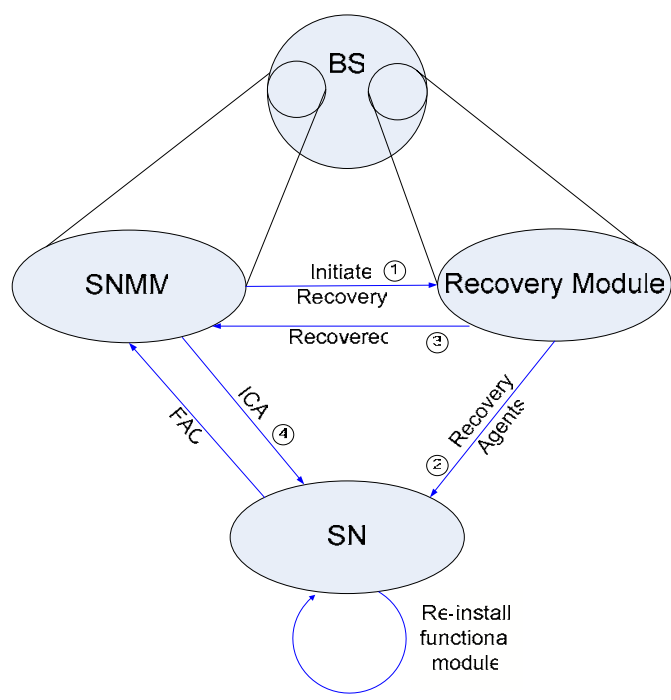

Figure 5: Recovery Process

\section{Recovery Process}

The recovery module generates the recovery agents, which carry the parts of functional module and install it on the sensor node. Once this recovery is complete, the control passes over to the SNMM, which then creates the ICA to check the integrity of the recovered sensor node.

Since this entire security framework is dependent on the stable and sane environment provided by the middleware layer, it becomes crucial to examine the sanity of the middleware. In our framework, the middleware is closely bound to the operating system and since the operating system is running in a monolithic environment, we make this safe assumption that for any modification of the middleware requires a restart of the entire sensor node.

Hence the problem of checking for a sane middleware is reduced to see if the sensor node has restarted from the time it was last checked. For this reason we have a basic counter process running in each sensor node. The incrementing counter algorithm is different for each sensor node. So even if the malicious entity gains knowledge of the counter process algorithm in one node it may not be able to use this intelligence to attack another node. The disadvantage of this approach is that it binds the sensor node with the base station with loose synchronization.

When the sensor node is restarted by the malicious entity, the process gets re-initialized and the counter is reset to its initial value. The ICA takes back the value of this counter to the base station, where it's checked for validity of the middleware validity.

\section{SECURITY AND OVERHEAD ANALYSIS}

The performance of a security framework depends on the confidence with which the framework provides the guarantee of thwarting the attack. In our model, any change in the functional module will be caught during the next integrity check. Given the design and simplicity in the working of the framework, we can say that, irrespective of the amount of change in functional module, the integrity checker agent will be able to identify the infringement with $100 \%$ confidence.

This infringement can be caused by two factors. One factor is because some malicious entities try to perform damaging function. The second is the malfunctioning of node due to any unforeseen circumstances. Irrespective of the cause of the change in the functional module, the actions taken remain the same.

The security and the uniqueness of the FAC generated for the functional module is guaranteed by the unique IV sent by the base station as part of the ICA. Since we are not passing any message along with the FAC or the IV, we have the liberty of selecting the length of IV to our convenience. In our case we would like to have a strong and longer, non-repetitive IV, since the message (functional module) over which the FAC is calculated remains the same each time.

Since only the base station will perform legitimate node reprogramming it will be aware of legit functional module changes in the sensor node. Hence the base station can accordingly generate the expected FAC and these changes in the sensor node will not be considered malicious in the integrity check process.

The bandwidth and the energy consumption are constant for each transmission because of the fixed size of the ICA. The total amount of the energy consumed and the bandwidth used will depend on the number of times the integrity check is issued. The transmission and reception of ICA can be considered equivalent to the transmission and reception of any 29 byte (max packet size) control packet. So the frequency of the integrity check will correspondingly increase the energy consumed. But the frequency of integrity checking $\left(\mathrm{F}_{\mathrm{IC}}\right)$ is important considering the fact that it is inversely proportional to the execution time of the malfunctioning node $\left(\mathrm{E}_{\mathrm{MN}}\right)$.

$$
F_{I C} \propto \frac{1}{E_{M N}}
$$

Hence the decision on the $\mathrm{F}_{\mathrm{IC}}$ depends on the rate at which the base-station receives the malfunction information about nodes. If the number of malfunctioning nodes increases over a period, then $\mathrm{F}_{\mathrm{IC}}$ needs to be increased.

Let the occurrence of the malfunctioning nodes in the network follows the Poisson distribution. The rate of occurrence of the malfunctioning nodes is $\mathrm{n}$ per minute. This implies that over a period of time $t$, we have $n * t$ malfunctioning nodes.

Suppose our network has 100 nodes, and the rate of malfunctioning is 2 nodes per minute. We check the integrity of the nodes every t minutes. Table 1 shows the distribution of the cumulative downtime of the nodes for different values of $t$ over a period of $1 \mathrm{hr}$. If all the nodes function properly, then we should have a total sensing time of 100 hours summed over all the nodes. We assume that once ICA is sent, the identification and the recovery of the node take a very small time comparatively.

Another factor is the amount of energy consumed for such an integrity check. The total energy consumed per integrity check is as follows

$$
E_{t o t}=R_{I C A}+E_{F A C}+S_{I C A}
$$


Where $\mathrm{E}_{\text {tot }}-$ Total energy spent by the $\mathrm{SN}$ / integrity check.

$\mathrm{R}_{\mathrm{ICA}}$ - Energy spent for receiving the ICA

$\mathrm{E}_{\mathrm{FAC}}-$ Energy spent to generate the FAC

$\mathrm{S}_{\mathrm{ICA}}$ - Energy required for transmitting the ICA

This $\mathrm{E}_{\text {tot }}$ can be assumed to be a constant value, since $\mathrm{R}_{\mathrm{ICA}}, \mathrm{E}_{\mathrm{FAC}}, \mathrm{S}_{\mathrm{ICA}}$ are all constant values, given the fact that size of ICA is fixed, and the energy required to generate the FAC is also fixed as the size of the functional module is a constant. It is a known fact in wireless networks that the amount of energy required to transmit 1 bit is approximately equivalent to execution of $800 \mathrm{CPU}$ cycles. Considering this, coupled with the fact that we do not transmit the encrypted functional module, but only the FAC, the overall energy consumption $\mathrm{E}_{\mathrm{tot}}$ will not be high.

Table 1: Distribution of cumulative downtime of network and the energy

\begin{tabular}{|c|c|c|c|c|}
\hline $\begin{array}{c}\text { Check } \\
\text { Freq }\end{array}$ & $\begin{array}{c}\text { Total Outage } \\
\text { (Minutes) }\end{array}$ & $\begin{array}{c}\% \\
\text { Outage }\end{array}$ & $\begin{array}{c}\text { Maximum } \\
\text { Outage } \\
\text { (Num nodes) }\end{array}$ & $\begin{array}{c}\text { Energy } \\
\text { Overhead/ } \\
\text { Node }\end{array}$ \\
\hline 5 & 360 & 6 & 10 & $12^{*} \mathrm{E}_{\text {tot }}$ \\
\hline 10 & 660 & 11 & 20 & $6^{*} \mathrm{E}_{\text {tot }}$ \\
\hline 30 & 930 & 15.5 & 60 & $2^{*} \mathrm{E}_{\text {tot }}$ \\
\hline 60 & 1830 & 30.5 & 100 & $\mathrm{E}_{\text {tot }}$ \\
\hline
\end{tabular}

This reasoning is in correspondence with the measurements by Karlof et al. in [10], in which they state that the increase in energy consumption comes from the transmission of the increased length packet and not from the encryption. In our case, this adds to our advantage because the small ICA to be transmitted back only contains the counter value and the FAC and no other data.

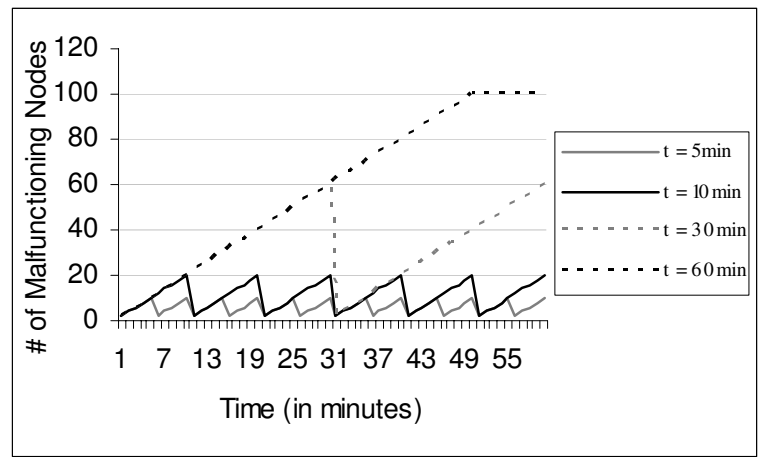

Figure 6: Malfunction Node Distribution

From Table 1 we see the amount of total sensing time decreases with decrease in the frequency of checking the integrity of the sensor node.

We do not take into consideration the amount of energy spent for recovering the node, because we presume any sensing functionality we get after recovering the node is an additional bonus, as compared to having a malfunctioning node.

Another important feature we may need to take into consideration is the maximum outage (maximum number of malfunctioning nodes) possible at any given instant of time. We see that when the frequency of integrity check is 60 minutes, there is a possibility that the entire network has started to malfunction before the recovery can be initiated. This can be seen from Figure 6 .

Considering all the factors discussed above, the base station may need to take different approaches under different scenarios. If the rate at which the nodes are malfunctioning is increasing, the base station may need to increase the frequency of integrity check. If this was not possible over all the nodes, then it becomes the responsibility of the base station to identify certain crucial node (example- boundary nodes or nodes whose death may split the sensor network into two) and increase the integrity check frequency on such nodes.

\section{RELATED WORK}

Because sensors' security is crucial for military applications, a lot of research has been devoted to this area. Most of the work (TinySec[10], SPIN[13], TinyPK[15], SERP[8]) is motivated to protect the integrity and confidentiality of transmission data by using encryption and authentication mechanisms. Different from this work, our work is focused on protecting sensor nodes against attacks initiated to break or alter sensors normal functionalities for malicious intent.

To protect sensors against physical attacks which completely destroy sensors' functionalities, Xuan et al. proposed in [16] that some nodes behave as sacrificial nodes by performing the function even with the knowledge of intruder lurking around, while other neighbor nodes can switch themselves off, thereby going undetected. Compared with [16], our proposed approach protects sensors against attacks, which reprogram sensors through radio signals for malicious purpose. Instead of causing a complete out of service, the malicious nodes try to disguise themselves by functioning as normal, while attacking the entire network sneakily.

To identify sensors that pretend to function as normal while having malicious intent, Srivastava et al. introduced reputation-based approach in RFSN [7]. Similar to Keynote [1] and Ebay [14], RFSN uses a trust-based system in which sensor nodes maintain reputation for each other. By maintaining this reputation they are able to identify incorrect information and discard it. To identify disguised malicious nodes, semantic analysis and pattern matching approach was proposed in [4] [3] [12] [2]. In [2] the authors define rules in the form of pushdown automata and check if this decries any security goal, which is similar to the method to check for vulnerabilities or bugs in the program codes. Both reputationbased approach and semantic-based approach usually have heavy overhead, which incurs large communications to collect data and intensive computations to analyze data. In contrast, our proposed mobile agent based approach use less intensive computation, which can be fully implemented on current MICA2 platform.

Besides detecting malicious nodes, our approach can also recover sensors' normal functionalities by mobile agents, which is first proposed to be applied to sensor networks in Agilla [5] [6]. While Agilla is a general node reprogramming technique for quick deployment of sensor nodes, our approach 
specially apply this technique to detect malicious sensors nodes and recover their normal functionalities.

\section{CONCLUSION AND FUTURE WORK}

In this paper, we propose a layered framework to protect sensors from malicious attacks. We clearly categorize the attacks into two types: attacks initiated through radio signals and physical attacks. Our proposed layered framework specially defends against the radio signal based attacks, which is more disguised and hazardous. Besides identifying the malicious nodes, our proposed solution can also recover the normal functionality of sensors, which has been altered by malicious radio signals. To the best of our knowledge, this work is first of its kind in the domain of sensor networks, wherein the recovery of node is considered. This framework is open and can be used as a basis for implementing other secure protocols and can be extended to provide security against other kind of attacks. As part of future work, we want to extend the framework to provide a distributed integrity checking mechanism in which the neighboring nodes can perform the required integrity check.

\section{REFERENCES}

[1] M. Blaze, J. Frigrnbaum, J. Ionnidis and A. Keromytis. RFC2704 - The keynote trust management system version 2. 1999.

[2] H. Chen and D. Wagner. MOPS: An infrastructure for examining security properties of software, In proceedings of the 9th ACM Conference on Computer and Communications Security, November 2002, pp. 235-244.

[3] M. Christodorescu and S. Jha. Static analysis of executables to detect malicious patterns. In proceedings of the $12^{\text {th }}$ USENIX Security Symposium (Security '03), pages 169-186. USENIX Association, Aug. 2003.

[4] M. Christodorescu, S. Jha, S. Seshia, D. Song, and R. Bryant. Semantics-aware malware detection. In proceedings of IEEE Symposium on Security and Privacy, Oakland, May 2005.
[5] C. Fok, G. Roman, C. Lu. Rapid development and flexible deployment of adaptive wireless sensor network applications, In proceedings of the 24th International Conference on Distributed Computing Systems (ICDCS'05), Columbus, Ohio, 2005.

[6] C. Fok, G. Roman, C. Lu. Mobile agent middleware for sensor networks: An application case study, In proceedings of the 4th International Conference on Information Processing in Sensor Networks (IPSN'05), Los Angeles, California, 2005

[7] S. Ganeriwal, M. B. Srivastava. Reputation-based framework for high integrity sensor networks, In proceedings of ACM Security for Ad-hoc and Sensor Networks (SASN 2004).

[8] S. Ganeriwal, R. Kumar, C. Han, S. Lee, M. Srivastava. Secure eventreporting protocol for sense-response applications. CENS Technical report. January 2005

[9] C. Karlof and D. Wagner. Secure routing in wireless sensor networks: Attacks and countermeasures, In proceedings of the First IEEE International Workshop on Sensor Network Protocols and Applications, May 2003.

[10] C. Karlof, N. Sastry, D. Wagner. TinySec: A link layer security architecture for wireless sensor networks, In proceedings of Sensys '04.

[11] D. Kotz, R. Gray. Mobile agents and future of internet, In proceedings of ACM Operating Systems Review 33(3), August 1999, pp. 7-13.

[12] C. Kruegel, W. Robertson and G. Vigna. Detecting kernel-level rootkits though binary analysis, In proceedings of the $20^{\text {th }}$ Annual Computer Security Applications Conference (ACSAC '04), Tuscon, AZ, Dec 2004.

[13] A. Perrig, R. Szewczyk, V. Wen, D. Culler, and J. Tygar. SPINS: Security protocols for sensor networks, In proceedings of the 7th ACM Mobile Computing and Networks (MobiCom '01), pp. 189-199, July 2001.

[14] P. Resnick, R. Zeckhauser. Trust among strangers in internet transactions: Empirical analysis of eBay's reputation system, In proceedings of NBER workshop on empirical studies of electronic commerce, 2000.

[15] R. Watro, D. Kong, S. F. Cuti, C. Gardiner, C. Lynn., and P. Kruus. TinyPK: Securing sensor networks with public key technology, In proceedings of the 2nd ACM Workshop on Security of Ad Hoc and Sensor Networks (Washington D.C., Oct. 2004), ACM Press, pp. 59-64.

[16] X. Wang, W. Gu, K. Schosek, S. Chellappan and D. Xuan, Lifetime optimization of sensor networks under physical attacks, In proceedings of IEEE International Conference on Communications (ICC), May 2005. 